\title{
State-Of-The-Art Laser-Diagnostic Measurement Techniques Applied To Multiphase Flows
}

\author{
Christos N. Markides* \\ Clean Energy Processes (CEP) Laboratory, Department of Chemical Engineering \\ Imperial College London, U.K. \\ * c.markides@imperial.ac.uk
}

\begin{abstract}
Multiphase, and in particular two-phase, flows are encountered in a broad range of settings, from highly complex isothermal flows in upstream and midstream oil-and-gas applications, to flows in the presence of heating or cooling and phase change such as in heat exchangers, condensers, evaporators, absorbers and reactors in diverse industrial processes across a wide range of scales.

Despite the numerous and well-performed experimental studies encountered in literature, only a limited number of studies relating to the simultaneous and spatiotemporal variations of the interfaces and underlying velocity and other scalar fields in these flows are currently available; a limitation linked inherently to the many challenges that arise when performing these measurements. Two-phase flows, in particular, present the experimentalist with a unique set of characteristics, including restricted (often sub-mm) fluid domains, moving and complex interfaces, and phases with large density or refractive index changes that render the extraction of reliable information challenging.

In this talk, we will present recent efforts to develop and apply a range of laser-based and other combined diagnostic techniques to a number of multiphase flows, both isothermal and in the presence of heat transfer, and extending to phase change processes (boiling and freezing). We will cover isothermal horizontal and inclined liquid-liquid flows, vertical gas-liquid flows and falling film flows, as well as diabatic flows such as heat falling films, and finally flows with phase change due to presence of heat transfer including pool boiling and droplet freezing. Important results from all of these efforts will be presented and new insights discussed.
\end{abstract}

